\title{
On a class of wreath products of hypergroups and association schemes
}

\author{
Rie Tanaka $\cdot$ Paul-Hermann Zieschang
}

Received: 28 June 2011 / Accepted: 4 May 2012 / Published online: 26 May 2012

(C) Springer Science+Business Media, LLC 2012

\begin{abstract}
We characterize finite hypergroups $S$ (in the sense of Frédéric Marty in Huitième Congres des Mathématiciens, pp. 45-59, 1934) satisfying $|p q|=1$ for any two elements $p$ and $q$ in $S$ with $p \neq q^{*}$ in terms of wreath products. The result applies to association schemes of finite valency and provides a corresponding characterization in scheme theory. For association schemes $S$ of finite valency satisfying the above condition, we provide a second characterization, a characterization in terms of the subconstituent algebra of $S$.
\end{abstract}

Keywords Groups · Association schemes · Hypergroups · Wreath products ·

Subconstituent algebras

Let $S$ be a set, and let $\mu$ be a map from $S \times S$ to the power set of $S$. For any two elements $p$ and $q$ of $S$, we write $p q$ instead of $\mu(p, q)$ and assume that $p q$ is not empty.

For any two nonempty subsets $P$ and $Q$ of $S$, we define the complex product $P Q$ to be the union of the sets $p q$ with $p \in P$ and $q \in Q$. If one of the two factors in a complex product consists of a single element, say $s$, we write $s$ instead of $\{s\}$ in that product.

Following (and generalizing) Frédéric Marty's terminology in [4], we call $S$ a hypergroup (with respect to $\mu$ ) if the following three conditions hold: ${ }^{1}$

\footnotetext{
${ }^{1}$ It is worth mentioning that the notion of a hypergroup arises in many different ways in the literature. For fairly complete overviews on the different definitions of hypergroups that have been given since Marty, we refer to [1] and [3]. The definition we use in the present note is almost Marty's original definition. The only difference is that we do not assume that $p q=q p$ for any two hypergroup elements $p$ and $q$.
}

This work was done while the first named author was visiting the University of Wisconsin-Madison. She would like to thank the department for its hospitality.

R. Tanaka $(\bowtie)$

Sendai, Japan

e-mail: hrie240@gmail.com

P.-H. Zieschang

Department of Mathematics, University of Texas at Brownsville, Brownsville, TX 78520, USA

e-mail: zieschang@utb.edu 
H1 For any three elements $p, q$, and $r$ in $S$, we have $p(q r)=(p q) r$.

H2 The set $S$ possesses an element $e$ such that $s e=\{s\}$ for each element $s$ in $S$.

H3 For each element $s$ in $S$, there exists an element $s^{*}$ in $S$ such that $p \in r q^{*}$ and $q \in p^{*} r$ for any three elements $p, q$, and $r$ in $S$ satisfying $r \in p q$.

Each association scheme satisfies the above three conditions $\mathrm{H} 1, \mathrm{H} 2$, and $\mathrm{H} 3$ with respect to its complex multiplication; cf. [8, Lemma 1.3.1], [8, Lemma 1.3.3(i)], and [8, Lemma 1.3.3(ii)]. Thus, hypergroups generalize association schemes. In particular, they generalize groups. ${ }^{2}$

It is not so much the fact itself that each association scheme (and, therefore, each group) is a hypergroup which caught our interest in hypergroups, more interesting is the observation that many proofs in scheme theory can be divided clearly into a part which uses only hypergroup theoretic arguments and a part in which one has to deal with the underlying set on which the association scheme is defined. A first systematic attempt to separate the hypergroup axioms from scheme theory was made in [9]. Condition (a) of Theorem A seems to be a natural question within the framework of [9].

The hypergroups which correspond to groups are the hypergroups $S$ in which $|p q|=1$ for any two elements $p$ and $q$ in $S{ }^{3}$ In the present article, we slightly weaken this latter condition by looking at hypergroups $S$ where $|p q|=1$ only for elements $p$ and $q$ in $S$ with $p \neq q^{*}$. We prove the following theorem.

Theorem A Let $S$ be a finite hypergroup. Then the following conditions are equivalent:

(a) For any two elements $p$ and $q$ in $S$ with $p \neq q^{*}$, we have $|p q|=1$.

(b) The hypergroup $S$ is a wreath product of finitely many finite hypergroups all of which are thin, are generated by an involution, or are generated by an idempotent element of $S$.

The definition of a wreath product of hypergroups will be given in Section 3. The definition of a thin hypergroup, the one of an involution, and the one of an idempotent element of a hypergroup will be given in Section 4.

Since each association scheme is a hypergroup with respect to its complex multiplication, Theorem A applies to association schemes. More specifically, we shall prove the following (which generalizes part of [6, Theorem 1]).

Theorem B Let $S$ be an association scheme of finite valency. Then the following conditions are equivalent.

(a) For any two elements $p$ and $q$ in $S$ with $p \neq q^{*}$, we have $|p q|=1$.

\footnotetext{
${ }^{2}$ We also should mention that the set of the distinguished basis elements of a table algebra in the sense of [2] is a finite hypergroup with respect to the multiplication introduced in [2, Definition 1.4]. Of course, the converse is not true. In fact, each hypergroup element $s$ satisfying $s s=\{s\}, s^{*} \neq s$, and $s^{*} s=\left\{1, s, s^{*}\right\}$ gives rise to a hypergroup with three elements which does not come from a table algebra.

${ }^{3}$ In Lemma 1.5, we shall see that these are exactly the hypergroups which contain "thin" elements only.
} 
(b) The scheme $S$ is a wreath product of finitely many finite schemes all of which are thin or generated by an involution.

(c) For each nonprimary irreducible module $W$ of the subconstituent algebra of $S$, we have $|\operatorname{Supp}(W)|=1$.

As far as scheme theoretic terminology and notation is concerned, we refer to [8]. The subconstituent algebra of an association scheme was introduced in [7]. We shall give its definition at the beginning of Section 6. Section 6 is also the place where we define the primary irreducible module of the subconstituent algebra and the set $\operatorname{Supp}(W)$.

The authors would like to thank the referees of this article for insightful comments and for several simplifications of our original arguments.

\section{Preliminaries on hypergroups}

In this section, the letter $S$ stands for a hypergroup.

The first three results of this section were first proven in [9]. They are [9, Lemma 1.1.1], [9, Lemma 1.1.2], and [9, Lemma 1.1.4(i), (ii)]. In order to keep this note self-contained, we repeat the (short) proofs of these lemmas here.

An element $e$ of $S$ which satisfies $s e=\{s\}$ for each element $s$ in $S$ is called a neutral element of $S$. Condition $\mathrm{H} 2$ guarantees the existence of neutral elements.

Lemma 1.1 Let $s$ be an element in $S$. Then the following hold:

(i) For each neutral element e of $S$, we have e $\in s^{*} s$.

(ii) We have $s^{* *}=s$.

(iii) For each neutral element e of $S$, we have es $=\{s\}$.

Proof (i) Let $e$ be a neutral element of $S$. Then, by definition, $s \in s e$. Thus, by H3, $e \in s^{*} s$.

(ii) From (i) we know that $e \in s^{*} s$. Thus, by $\mathrm{H} 3, s \in s^{* *} e$. On the other hand, by $\mathrm{H} 2, s^{* *} e=\left\{s^{* *}\right\}$. Thus, $s \in\left\{s^{* *}\right\}$, and that means that $s^{* *}=s$.

(iii) Let $r$ be an element in es. Then, by $\mathrm{H} 3, e \in r s^{*}$. A second application of H3 yields $s^{*} \in r^{*} e$. On the other hand, by H2, $r^{*} e=\left\{r^{*}\right\}$. Thus, $s^{*} \in\left\{r^{*}\right\}$, and that means that $r^{*}=s^{*}$. Thus, by (ii), $r=r^{* *}=s^{* *}=s$.

Lemma 1.2 The hypergroup S possesses exactly one neutral element.

Proof Let $c$ and $d$ be neutral elements of $S$. Then, by Lemma 1.1(iii), $\{c\}=c d=\{d\}$. It follows that $c=d$.

For the remainder of this note, the uniquely determined neutral element of a hypergroup will be denoted by 1 . Looking at $\mathrm{H} 2$ and $\mathrm{H} 3$, one easily verifies that $1^{*}=1$. 
Lemma 1.3 Let $p$ and $q$ be elements in S. Then the following hold:

(i) We have $1 \in p q$ if and only if $p=q^{*}$.

(ii) Let $r$ be an element in $S$ satisfying $r \in p q$. Then $r^{*} \in q^{*} p^{*}$.

Proof (i) Assume that $1 \in p q$. Then, by H3, $p \in 1 \cdot q^{*}$. On the other hand, by Lemma 1.1(iii), $1 \cdot q^{*}=\left\{q^{*}\right\}$. Thus, $p \in\left\{q^{*}\right\}$, and that means that $p=q^{*}$.

Conversely, assume that $p=q^{*}$. Then, by Lemma 1.1(i), $1 \in q^{*} q=p q$.

(ii) Applying $\mathrm{H} 3$ three times, we obtain from $r \in p q$ first $p \in r q^{*}$, then $q^{*} \in r^{*} p$, and, finally, $r^{*} \in q^{*} p^{*}$.

For each element $s$ of $S$, we define

$$
T_{s}:=\{r \in S \mid s r=\{s\}\} .
$$

Note that $T_{1}=\{1\}$.

Lemma 1.4 Let $s$ be an element in S. Then the following hold:

(i) Assume that $s \neq 1$. Then $T_{s} \subseteq s^{*} s \backslash\left\{s^{*}\right\}$.

(ii) Let $p$ and $q$ be elements in $T_{s}$. Then $p q \subseteq T_{s}$.

Proof (i) Let $r$ be an element in $T_{s}$. Then, by definition, $s r=\{s\}$. In particular, $s \in s r$. Thus, by H3, $r \in s^{*} s$.

Assume that $s^{*} \in T_{s}$. Then, by definition, $s s^{*}=\{s\}$. On the other hand, by Lemma 1.1(i), (ii), $1 \in s s^{*}$. Thus, $s=1$, contradicting our choice of $s$.

(ii) Since $p \in T_{s}, s p=\{s\}$. Similarly, as $q \in T_{s}, s q=\{s\}$. Thus, $s p q=\{s\}$. It follows that $s r=\{s\}$ for each element $r$ in $p q$.

Recall from Lemma 1.1(i) that $1 \in s^{*} s$ for each element $s$ in $S$. An element $s$ of $S$ is called thin if $s^{*} s=\{1\}$. The set of all thin elements of $S$ will be denoted by $O_{\vartheta}(S)$.

Lemma 1.5 For any two elements $p$ and $q$ in $O_{\vartheta}(S)$, there exists an element $r$ in $O_{\vartheta}(S)$ such that $p q=\{r\}$.

Proof Let $p$ and $q$ be elements of $O_{\vartheta}(S)$. Then $p^{*} p=\{1\}$ and $q^{*} q=\{1\}$.

Let $r$ and $s$ be elements in $p q$. Then, referring to Lemma 1.3(ii), we obtain

$$
r^{*} s \subseteq q^{*} p^{*} p q \subseteq q^{*} q=\{1\} .
$$

Thus, by Lemma 1.3(i), $r^{*}=s^{*}$. It follows that $r=s$; cf. Lemma 1.1(ii). Thus, as $r^{*} s \subseteq\{1\}, r^{*} r \subseteq\{1\}$, and that means that $r \in O_{\vartheta}(S)$.

\section{Closed subsets of hypergroups}

In this section, the letter $S$ stands for a hypergroup.

A nonempty subset $R$ of $S$ is called closed if $p^{*} q \subseteq R$ for any two elements $p$ and $q$ in $R$.

Note that $\{1\}$ and $S$ are closed and that intersections of closed subsets are closed. 
Lemma 2.1 Let $T$ and $U$ be closed subsets of $S$. Then the following hold:

(i) For any two elements $p$ and $q$ in $S$ with $p \in T q U$, we have $T q U \subseteq T p U$.

(ii) The set $\{T s U \mid s \in S\}$ is a partition on $S$.

Proof (i) Let $p$ and $q$ be elements in $S$, and assume that $p \in T q U$. Then there exist elements $t$ in $T$ and $u$ in $U$ such that $p \in t q u$. Thus, $t q$ possesses an element $w$ such that $p \in w u$.

From $w \in t q$ we obtain $q \in t^{*} w$; cf. H3. Similarly, as $p \in w u, w \in p u^{*}$. Thus, as $T$ and $U$ are assumed to be closed,

$$
q \in t^{*} w \subseteq t^{*} p u^{*} \subseteq T p U .
$$

It follows that $T q U \subseteq T p U$.

(ii) For each element $s$ in $S$, we have $s \in T s U$. Thus, $S$ is equal to the union of the sets $T s U$ with $s \in S$.

Let $p$ and $q$ be elements in $S$, assume that $T p U \cap T q U$ is not empty, and let $r$ be an element in $T p U \cap T q U$. Since $r \in T p U, \operatorname{Tr} U \subseteq T p U$. From $r \in T p U$ we also obtain $T p U \subseteq \operatorname{Tr} U$; cf. (i). Thus, $\operatorname{Tp} U=\operatorname{Tr} U$. Similarly, $\operatorname{Tq} U=\operatorname{Tr} U$, so that $T p U=T q U$.

Lemma 2.2 Let $T$ be a closed subset of $S$. Let $p, p^{\prime}, q$, and $q^{\prime}$ be elements in $S$, and assume that $T p T=T p^{\prime} T$ and $T q T=T q^{\prime} T$. Then $\{\operatorname{Tr} T \mid r \in p T q\}=\{\operatorname{Tr} T \mid r \in$ $\left.p^{\prime} T q^{\prime}\right\}$.

Proof Let $s$ be an element in $S$, and assume that $T s T \in\{\operatorname{Tr} T \mid r \in p T q\}$. Then there exists an element $r$ in $p T q$ such that $\operatorname{Ts} T=\operatorname{Tr} T$. It follows that

$$
s \in T r T \subseteq T p T q T=T p^{\prime} T q^{\prime} T .
$$

Thus, there exists an element $r^{\prime}$ in $p^{\prime} T q^{\prime}$ such that $s \in T r^{\prime} T$. From $s \in T r^{\prime} T$ we obtain $T s T=T r^{\prime} T$; cf. Lemma 2.1(ii). Thus, as $r^{\prime} \in p^{\prime} T q^{\prime}, T s T \in\left\{T r T \mid r \in p^{\prime} T q^{\prime}\right\}$.

So far, we have shown that $\{\operatorname{Tr} T \mid r \in p T q\} \subseteq\left\{\operatorname{Tr} T \mid r \in p^{\prime} \operatorname{Tq^{\prime }}\right\}$. That $\{\operatorname{Tr} T \mid$ $\left.r \in p^{\prime} T q^{\prime}\right\} \subseteq\{\operatorname{Tr} T \mid r \in p T q\}$ follows similarly.

Let $T$ be a closed subset of $S$. For each element $s$ in $S$, we define

$$
s^{T}:=T s T,
$$

for each closed subset $U$ of $S$ with $T \subseteq U$, we set

$$
U / / T:=\left\{u^{T} \mid u \in U\right\},
$$

and, for any two elements $p$ and $q$ of $S$, we define

$$
\mu_{T}\left(p^{T}, q^{T}\right):=\left\{r^{T} \mid r \in p T q\right\} .
$$

Then, by Lemma 2.2, $\mu_{T}$ is a map from $S / / T \times S / / T$ to the power set of $S / / T$. In the following, we shall write $p^{T} q^{T}$ instead of $\mu_{T}\left(p^{T}, q^{T}\right)$ whenever $p$ and $q$ are elements in $S$. Moreover, for each element $s$ in $S$, we define $\left(s^{T}\right)^{*}:=\left(s^{*}\right)^{T}$. 
Lemma 2.3 Let $T$ be a closed subset of $S$. Then $S / / T$ is a hypergroup (with respect to $\left.\mu_{T}\right)$.

Proof From H1 one easily obtains $p^{T}\left(q^{T} r^{T}\right)=\left(p^{T} q^{T}\right) r^{T}$ for any three elements $p$, $q$, and $r$ in $S$.

For each element $s$ in $S$, we obviously have $s^{T} 1^{T}=\left\{s^{T}\right\}$.

Let $p, q$, and $r$ be elements in $S$, and assume that $r^{T} \in p^{T} q^{T}$. We first show that $p^{T} \in r^{T}\left(q^{T}\right)^{*}$.

From $r^{T} \in p^{T} q^{T}$ we obtain an element $r^{\prime}$ in $p T q$ such that $r^{T}=r^{\prime T}$.

From $r^{\prime} \in p T q$ we obtain an element $t$ in $T$ such that $r^{\prime} \in p t q$. From $r^{\prime} \in p t q$ we obtain $p \in r^{\prime} q^{*} t^{*}$; cf. H3 and Lemma 1.3(ii). Thus, $r^{\prime} q^{*}$ possesses an element $p^{\prime}$ such that $p \in p^{\prime} t^{*}$.

From $p^{\prime} \in r^{\prime} q^{*}$ we obtain $p^{\prime} \in r^{\prime} T q^{*}$. Thus, by definition, $p^{\prime T} \in r^{\prime T}\left(q^{T}\right)^{*}$. From $p \in p^{\prime} t^{*}$ we obtain $T p T=T p^{\prime} T$; cf. Lemma 2.1(ii). Thus, $p^{T}=p^{\prime T}$. It follows that $p^{T} \in r^{\prime T}\left(q^{T}\right)^{*}$. Thus, as $r^{T}=r^{\prime T}, p^{T} \in r^{T}\left(q^{T}\right)^{*}$.

That $q^{T} \in\left(p^{T}\right)^{*} r^{T}$ follows similarly.

Let $R$ be a subset of $S$. We define $\langle R\rangle$ to be the intersection of all closed subsets of $S$ which contain $R$ (as a subset). If $R=\{r\}$ for some element $r$ in $S$, we write $\langle r\rangle$ instead of $\langle R\rangle$. Note that $\langle R\rangle$ is closed. We call $\langle R\rangle$ the closed subset generated by $R$. We define $R^{*}$ to be the set of all elements $r^{*}$ with $r \in R$. If $R$ is not empty, we set $R^{0}:=\{1\}$ and define inductively $R^{n}:=R^{n-1} R$ for each positive integer $n$. The following lemma is [9, Lemma 1.6.1].

Lemma 2.4 Let $R$ be a nonempty subset of $S$. Then $\langle R\rangle$ is equal to the union of the sets $\left(R^{*} \cup R\right)^{n}$ with $n$ a nonnegative integer.

Proof Set $P:=R^{*} \cup R$ and define $Q$ to be the union of the sets $P^{n}$ with $n$ a nonnegative integer. We have to show that $\langle R\rangle=Q$.

Since $P^{*}=P,\left(P^{n}\right)^{*}=P^{n}$ for each nonnegative integer $n$; cf. Lemma 1.3(ii). Thus, for any two nonnegative integers $k$ and $l$,

$$
\left(P^{k}\right)^{*} P^{l}=P^{k} P^{l}=P^{k+l} \subseteq Q .
$$

It follows that $Q$ is closed. Thus, as $R \subseteq Q,\langle R\rangle \subseteq Q$.

Conversely, for each nonnegative integer $n$, we have

$$
P^{n} \subseteq\langle P\rangle^{n} \subseteq\langle P\rangle=\langle R\rangle
$$

Therefore, $Q \subseteq\langle R\rangle$.

Lemma 2.5 Let $s$ be an element in S. Then the following hold.

(i) Let $R$ be a nonempty subset of $T_{s}$ satisfying $R^{*} \subseteq T_{s}$. Then $\langle R\rangle \subseteq T_{s}$.

(ii) Assume that $\left(T_{S}\right)^{*}=T_{S}$. Then $T_{S}$ is a closed subset of $S$. 
Proof (i) Let $n$ be a positive integer, and let $s_{1}, \ldots, s_{n}$ be elements in $R^{*} \cup R$. Then, by Lemma 1.4(ii), $s_{1} \cdots s_{n} \subseteq T_{s}$. Thus, the claim follows from Lemma 2.4.

(ii) Setting $R=T_{s}$ in (i), this follows from (i).

Lemma 2.6 Assume $S$ to be finite. Then $O_{\vartheta}(S)$ is a closed subset of $S$.

Proof Let $s$ be an element in $O_{\vartheta}(S)$. Considering Lemma 1.5, we shall be done if we succeed in showing that $s^{*} \in O_{\vartheta}(S)$.

From Lemma 1.5 we obtain $\left|s^{n}\right|=1$ for each positive integer $n$. Thus, as $S$ is assumed to be a finite set, there exist positive integers $k$ and $l$ such that $k \leq l-1$ and $s^{k}=s^{l}$. Let $p$ denote the uniquely determined element in $s^{l}$. Then, as $s^{k}=s^{l}$, $s^{k}=\{p\}$. Thus, by Lemma 1.3(ii), $\left(s^{*}\right)^{k}=\left\{p^{*}\right\}$. It follows that

$$
1 \in p^{*} p=\left(s^{*}\right)^{k} s^{l}=\left(s^{*}\right)^{k} s^{k} s^{l-k}=s^{l-k}=s^{l-k-1} s .
$$

Thus, $s^{l-k-1}$ possesses an element $q$ such that $1 \in q s$. Thus, by Lemma 1.3(i), $q=$ $s^{*}$. Thus, as $q \in s^{l-k-1}$,

$$
s^{*} \in s^{l-k-1} \subseteq O_{\vartheta}(S)
$$

cf. Lemma 1.5.

\section{Homomorphisms of hypergroups}

Let $S$ and $S^{\prime}$ be hypergroups. A map $\phi$ from $S$ to $S^{\prime}$ is called a homomorphism if $\phi(1)=1$ and

$$
\phi(p q)=\phi(p) \phi(q)
$$

for any two elements $p$ and $q$ in $S$.

Lemma 3.1 Let $S$ and $S^{\prime}$ be hypergroups, let $\phi$ be a homomorphism from $S$ to $S^{\prime}$, and let $s$ be an element in $S$. Then $\phi\left(s^{*}\right)=\phi(s)^{*}$.

Proof By definition, $\phi(1)=1$. On the other hand, by Lemma 1.1(i), $1 \in s^{*} s$. Thus,

$$
1=\phi(1) \in \phi\left(s^{*} s\right)=\phi\left(s^{*}\right) \phi(s) .
$$

Thus, by Lemma 1.3(i), $\phi\left(s^{*}\right)=\phi(s)^{*}$.

Let $S$ and $S^{\prime}$ be hypergroups, and let $\phi$ be a homomorphism from $S$ to $S^{\prime}$. We define

$$
\operatorname{ker}(\phi):=\{s \in S \mid \phi(s)=1\}
$$

and call this set the kernel of $\phi$.

Lemma 3.2 Let $S$ and $S^{\prime}$ be hypergroups, let $\phi$ be a homomorphism from $S$ to $S^{\prime}$, and set $T:=\operatorname{ker}(\phi)$. Then we have $p \in q T$ for any two elements $p$ and $q$ in $S$ satisfying $\phi(p)=\phi(q)$. 
Proof Let $p$ and $q$ be elements in $S$, and assume that $\phi(p)=\phi(q)$. Then

$$
1 \in \phi(q)^{*} \phi(p)=\phi\left(q^{*}\right) \phi(p)=\phi\left(q^{*} p\right)
$$

cf. Lemmas 1.3(i) and 3.1. Thus, $q^{*} p$ possesses an element $t$ such that $\phi(t)=1$. From $t \in q^{*} p$ we obtain $p \in q t$. From $\phi(t)=1$ we obtain $t \in T$. Thus, $p \in q T$.

A closed subset $T$ of a hypergroup $S$ will be called normal in $S$ if $T s \subseteq s T$ for each element $s$ in $S .^{4}$

A bijective homomorphism is called an isomorphism.

If there exists an isomorphism from a hypergroup $S$ to a hypergroup $S^{\prime}$, we say that $S$ and $S^{\prime}$ are isomorphic, and we indicate this by writing $S \cong S^{\prime}$.

In the following theorem, we shall write $\operatorname{im}(\phi)$ to denote the image of the map $\phi$.

Theorem 3.3 Let $S$ and $S^{\prime}$ be hypergroups, and let $\phi$ be a homomorphism from $S$ to $S^{\prime}$. Then the following hold:

(i) The set $\operatorname{ker}(\phi)$ is a normal closed subset of $S$.

(ii) The set $\mathrm{im}(\phi)$ is a closed subset of $S^{\prime}$.

(iii) We have $S / / \operatorname{ker}(\phi) \cong \operatorname{im}(\phi)$.

Proof (i) We first prove that $\operatorname{ker}(\phi)$ is closed. In order to do so, we fix elements $p$ and $q$ in $\operatorname{ker}(\phi)$ and an element $r$ in $p^{*} q$. We have to show that $r \in \operatorname{ker}(\phi)$.

From $p \in \operatorname{ker}(\phi)$ we obtain $\phi(p)=1$. Similarly, as $q \in \operatorname{ker}(\phi), \phi(q)=1$. Thus, referring to Lemma 3.1, we obtain

$$
\phi(r) \in \phi\left(p^{*} q\right)=\phi\left(p^{*}\right) \phi(q)=\phi(p)^{*} \phi(q)=1^{*} 1=\{1\} .
$$

It follows that $\phi(r)=1$, and that means that $r \in \operatorname{ker}(\phi)$.

Now we prove that $\operatorname{ker}(\phi)$ is normal in $S$. In order to do so, we set $T:=\operatorname{ker}(\phi)$. Let $t$ be an element in $T$, and let $s$ be an element in $S$. We have to show that $t s \subseteq s T$.

Let $r$ be an element in $t s$. Then

$$
\phi(r) \in \phi(t s)=\phi(t) \phi(s)=1 \cdot \phi(s)=\{\phi(s)\} .
$$

Thus, $\phi(r)=\phi(s)$. Thus, by Lemma 3.2, $r \in s T$.

(ii) Let $v$ and $w$ be elements in $\operatorname{im}(\phi)$. We have to show that $v^{*} w \subseteq \operatorname{im}(\phi)$.

Since $v \in \operatorname{im}(\phi), S$ possesses an element $p$ such that $\phi(p)=v$. Similarly, as $w \in$ $\operatorname{im}(\phi), S$ possesses an element $q$ such that $\phi(q)=w$. Thus, by Lemma 3.1,

$$
v^{*} w=\phi(p)^{*} \phi(q)=\phi\left(p^{*}\right) \phi(q)=\phi\left(p^{*} q\right) \subseteq \operatorname{im}(\phi) .
$$

(iii) Define $T:=\operatorname{ker}(\phi)$.

\footnotetext{
${ }^{4}$ Let $T$ be a normal closed subset of a hypergroup $S$, and let $s$ be an element in $S$. Then $T s^{*} \subseteq s^{*} T$. Thus, by Lemma 1.3(ii), $s T \subseteq T s$. On the other hand, as $T$ is assumed to be normal in $S, T s \subseteq s T$. It follows that $T s=s T$.
} 
We first claim that, for any two elements $p$ and $q$ in $S, p^{T}=q^{T}$ if and only if $\phi(p)=\phi(q)$.

Assume first that $p^{T}=q^{T}$. Then, as $T$ is normal in $S, p \in q T$, so that $p \in q t$ for some element $t$ in $T$. Thus,

$$
\phi(p) \in \phi(q t)=\phi(q) \phi(t)=\phi(q) \cdot 1=\{\phi(q)\} .
$$

It follows that $\phi(p)=\phi(q)$. Conversely, if $\phi(p)=\phi(q)$, then $p \in q T$; cf. Lemma 3.2. Thus, $T p T=T q T$, and that means that $p^{T}=q^{T}$.

Now we define $\psi\left(s^{T}\right):=\phi(s)$ for each element $s$ in $S$. Then, as $p^{T}=q^{T}$ if and only if $\phi(p)=\phi(q)$ for any two elements $p$ and $q$ in $S, \psi$ is an injective map from $S / / T$ to $\operatorname{im}(\phi)$. The definition of $\psi$ also implies that $\psi$ is surjective.

In order to show that $\psi$ is a homomorphism, we pick elements $p$ and $q$ in $S$. Then

$$
\begin{aligned}
\psi\left(p^{T} q^{T}\right) & =\psi\left(\left\{r^{T} \mid r \in p T q\right\}\right) \\
& =\left\{\psi\left(r^{T}\right) \mid r \in p T q\right\}=\{\phi(r) \mid r \in p T q\} .
\end{aligned}
$$

For each element $r$ in $p T q$, there exists an element $t$ in $T$ such that $r \in p t q$, and from $r \in p t q$ we obtain

$$
\phi(r) \in \phi(p t q)=\phi(p) \phi(t) \phi(q)=\phi(p) \cdot 1 \cdot \phi(q)=\phi(p q) .
$$

Thus,

$$
\psi\left(p^{T} q^{T}\right)=\phi(p q)=\phi(p) \phi(q)=\psi\left(p^{T}\right) \psi\left(q^{T}\right),
$$

and we are done.

Theorem 3.4 Let $S$ be a hypergroup, let $T$ and $U$ be closed subsets of $S$, and assume that $T \subseteq U$. Then the following hold:

(i) The set $U / / T$ is closed in $S / / T$.

(ii) Assume that $U$ is normal in $S$. Then $(S / / T) / /(U / / T) \cong S / / U$.

Proof (i) Let $p$ and $q$ be elements in $U$. We have to show that $\left(p^{T}\right)^{*} q^{T} \subseteq U / / T$.

Let $r$ be an element in $S$, and assume that $r^{T} \in\left(p^{T}\right)^{*} q^{T}$. Then, by definition, $r \in T p^{*} T q T \subseteq U$. It follows that $r^{T} \in U / / T$.

(ii) For any two elements $p$ and $q$ in $S$ with $T p T=T q T$, we have

$$
U p U=(U T) p(T U)=U(T p T) U=U(T q T) U=(U T) q(T U)=U q U .
$$

Thus, setting $\phi\left(s^{T}\right):=s^{U}$ for each element $s$ in $S, \phi$ is a surjective map from $S / / T$ to $S / / U$.

Note also that

$$
\phi\left(p^{T} q^{T}\right)=\phi\left(\left\{r^{T} \mid r \in p T q\right\}\right)=\left\{r^{U} \mid r \in p T q\right\}
$$

and

$$
\phi\left(p^{T}\right) \phi\left(q^{T}\right)=p^{U} q^{U}=\left\{r^{U} \mid r \in p U q\right\}
$$


for any two elements $p$ and $q$ in $S$. Thus, as $U$ is normal in $S$,

$$
\phi\left(p^{T} q^{T}\right)=\phi\left(p^{T}\right) \phi\left(q^{T}\right)
$$

for any two elements $p$ and $q$ in $S$.

Note finally that, for each element $s$ in $S, \phi\left(s^{T}\right)=1^{U}$ if and only if $s \in U$. Thus, $\operatorname{ker}(\phi)=U / / T$, and the claim follows from Theorem 3.3(iii).

Lemma 3.5 Let $S$ be a hypergroup, let $T$ be a closed subset of $S$, and assume that $T \subseteq T_{s}$ for each element $s$ in $S \backslash T$. Then $T$ is normal in $S$.

Proof For each element $s$ in $T$, we have $T s=T=s T$.

Let $s$ be an element in $S \backslash T$. Then $T \subseteq T_{S}$ by hypothesis. Thus, by definition $s T=\{s\}$.

Since $T$ is assumed to be closed, we obtain from $s \in S \backslash T$ also that $s^{*} \in S \backslash T$. Thus, $T \subseteq T_{s^{*}}$, and that means that $s^{*} T=\left\{s^{*}\right\}$. From this we obtain $T s=\{s\}$; cf. Lemma 1.3(ii). Thus, as $s T=\{s\}, T s=s T$.

Let $S_{1}$ and $S_{2}$ be hypergroups.

A hypergroup $S$ is said to be a wreath product of $S_{1}$ and $S_{2}$ if it possesses a closed subset $T$ such that

$$
T \cong S_{1}, \quad S / / T \cong S_{2},
$$

and $T \subseteq T_{S}$ for each element $s$ in $S \backslash T$.

If a hypergroup $S$ is a wreath product of $S_{1}$ and $S_{2}$, we write $S=S_{1}$ 乙 $S_{2}$.

Note that, by Lemma 3.5, the closed subset $T$ that arises in the definition of a wreath product $S$ is normal in $S$.

We also notice that a wreath product of association schemes in the sense of [5] is always a wreath product of hypergroups with respect to its complex multiplication. Conversely, if an association scheme $S$ is a wreath product of association schemes $S_{1}$ and $S_{2}$ with respect to its complex multiplication, and if $S_{1}$ is uniquely determined by its structure constants, then $S$ is a wreath product of the association schemes $S_{1}$ and $S_{2}$ in the sense of [5].

We shall now prove that wreath multiplication is associative.

Theorem 3.6 For any three hypergroups $S_{1}, S_{2}$, and $S_{3}$, we have $\left(S_{1}\right.$ 2 $\left.S_{2}\right)$ ? $S_{3}=$ $S_{1}$ 乙 $\left(S_{2}\right.$ 乙 $\left.S_{3}\right)$.

Proof Set $S:=\left(S_{1} 2 S_{2}\right)$ \ $S_{3}$. Then, by definition, $S$ possesses a closed subset $U$ such that

$$
U \cong S_{1} 2 S_{2}, \quad S / / U \cong S_{3}
$$

and $U \subseteq T_{s}$ for each element $s$ in $S \backslash U$. Since $U \cong S_{1} 2 S_{2}, U$ possesses a closed subset $T$ such that

$$
T \cong S_{1}, \quad U / / T \cong S_{2},
$$

and $T \subseteq T_{u}$ for each element $u$ in $U \backslash T$. 
Since $T \subseteq U$, we have $T \subseteq T_{S}$ for each element $s$ in $S \backslash T$. Thus, as $T \cong S_{1}$, we shall be done if we succeed in showing that $S / / T \cong S_{2}$ ? $S_{3}$.

We saw already that $U / / T \cong S_{2}$. Moreover, by Theorem 3.4(ii), $(S / / T) / /(U / / T) \cong$ $S / / U \cong S_{3}$. (Recall that, by Lemma 3.5, $U$ is normal in $S$.)

Let $s$ be an element in $S \backslash U$, and let $u$ be an element in $U$. Then, by definition,

$$
s^{T} u^{T}=\left\{r^{T} \mid r \in s T u\right\} .
$$

Since $s \in S \backslash U, U \subseteq T_{s}$. Thus, $s T u \subseteq s U=\{s\}$. It follows that $s^{T} u^{T}=\left\{s^{T}\right\}$. Thus, as $u$ has been chosen arbitrarily in $U$, we have shown that $U / / T \subseteq T_{S^{T}}$.

\section{Proof of Theorem A}

In this section, the letter $S$ stands for a hypergroup.

Recall that condition (a) of Theorem A says that $|p q|=1$ for any two elements $p$ and $q$ in $S$ with $p \neq q^{*}$. Within this section, we shall always mean condition (a) of Theorem A if we speak about condition (a).

Lemma 4.1 Assume that $S$ satisfies condition (a), and let $T$ be a normal closed subset of $S$. Then $S / / T$ satisfies condition (a).

Proof Let $p$ and $q$ be elements in $S$, and assume that $p^{T} \neq\left(q^{T}\right)^{*}$. Then $p \neq q^{*}$. Thus, as we assume that $S$ satisfies condition (a), there exists an element $s$ in $S$ such that $p q=\{s\}$. Thus, as $T$ is assumed to be normal in $S$,

$$
p^{T} q^{T}=\left\{r^{T} \mid r \in p T q\right\}=\left\{s^{T}\right\}
$$

so that $\left|p^{T} q^{T}\right|=1$.

Lemma 4.2 Assume that $S$ is finite and satisfies condition (a). Then $O_{\vartheta}(S) \subseteq T_{S}$ for each element $s$ in $S \backslash O_{\vartheta}(S)$.

Proof Set $T:=O_{\vartheta}(S)$, let $s$ be an element in $S \backslash T$, and let $t$ be an element in $T$. We have to show that $t \in T_{S}$, and that means that $s t=\{s\}$.

Since $S$ is assumed to be finite, $T$ is closed; cf. Lemma 2.6. Thus, as $t \in T, t^{*} \in T$.

Since $s \in S \backslash T$ and $t^{*} \in T, s \neq t^{*}$. Thus, as $S$ is assumed to satisfy condition (a), $s t=\{r\}$ for some element $r$ in $S$.

Assume, by way of contradiction, that $r \neq s$. Then $s^{*} \neq r^{*}$. Thus, as $S$ is assumed to satisfy condition (a), $\left|s^{*} r\right|=1$. Thus, as $t \in s^{*} r, s^{*} r=\{t\}$. Thus, as $s t=\{r\}$, $s^{*} s t=\{t\}$. It follows that

$$
s^{*} s=s^{*} s t t^{*}=t t^{*}=\{1\}
$$

recall that $t^{*} \in T$. Thus, by definition, $s \in T$, contradiction.

Lemma 4.3 Assume that $S$ satisfies condition (a), let $t$ be an element of $S$, and let $S$ be an element in $S \backslash\langle t\rangle$. Then the following hold: 
(i) We have $t^{*} t \subseteq T_{s}$.

(ii) Assume that $t \in t t$. Then $\langle t\rangle \subseteq T_{s}$.

Proof (i) Since $s \in S \backslash\langle t\rangle, s \neq t$. Thus, as $S$ is assumed to satisfy condition (a), $s t^{*}=\{r\}$ for some element $r$ in $S$.

From $r \in s t^{*}$ we obtain $s \in r t$; cf. H3. If $r=t^{*}, s \in t^{*} t \subseteq\langle t\rangle$, contradiction. Thus, $r \neq t^{*}$, so that $r t=\{s\}$.

From $s t^{*}=\{r\}$ and $r t=\{s\}$ we obtain $s t^{*} t=\{s\}$. Thus, $t^{*} t \subseteq T_{s}$.

(ii) We are assuming that $t \in t t$. Thus, $t \in t^{*} t$ and $t^{*} \in t^{*} t$. Thus, by (i), $t \in T_{s}$ and $t^{*} \in T_{s}$. Thus, by Lemma 2.5(i), $\langle t\rangle \subseteq T_{s}$.

Lemma 4.4 Assume that $S$ satisfies condition (a), and let $s$ be an element in $S \backslash\{1\}$. Then $T_{s}=s^{*} s \backslash\left\{s^{*}\right\}$.

Proof From Lemma 1.4(i) we know that $T_{s} \subseteq s^{*} s \backslash\left\{s^{*}\right\}$. So it remains to show that $s^{*} s \backslash\left\{s^{*}\right\} \subseteq T_{s}$.

Let $r$ be an element in $s^{*} \backslash \backslash\left\{s^{*}\right\}$. From $r \neq s^{*}$ we obtain $s \neq r^{*}$. Thus, as $S$ is assumed to satisfy condition (a), $|s r|=1$. On the other hand, as $r \in s^{*} s, s \in s r$; cf. H3. Thus, $s r=\{s\}$, and that means that $r \in T_{s}$.

Lemma 4.5 Assume that $S$ satisfies condition (a), let $s$ be an element in $S$, and assume that $s \notin s s$. Then the following hold.

(i) We have $T_{s}=s^{*} s$.

(ii) The set $T_{S}$ is a closed subset of $S$.

Proof (i) We are assuming that $s \notin s s$. Thus, by H3, $s \notin s s^{*}$. A second application of H3 now yields $s^{*} \notin s^{*} s$. Thus, the claim follows from Lemma 4.4.

(ii) From (i) we know that $T_{s}=s^{*} s$, from Lemma 1.3(ii) that $\left(s^{*} s\right)^{*}=s^{*} s$. Thus, $\left(T_{S}\right)^{*}=T_{s}$, so that the claim follows from Lemma 2.5(ii).

The hypergroup $S$ is called primitive if $S$ has exactly two closed subsets (which then must be $\{1\}$ and $S$.) It is called thin if each of its elements is thin.

Lemma 4.6 Assume that $S$ is primitive and satisfies condition (a). Assume further that $S$ is finite and that $S$ possesses an element s such that $s \notin s s$. Then $S$ is thin.

Proof We are assuming that $S$ possesses an element $s$ such that $s \notin s s$. Thus, by the definition of $T_{s}, s \notin T_{s}$. In particular, $T_{s} \neq S$.

On the other hand, according to Lemma 4.5(ii), $T_{S}$ is closed. Thus, as $S$ is assumed to be primitive, we must have $T_{s}=\{1\}$. Thus, by Lemma 4.5(i), $s^{*} s=\{1\}$, and that means that $s \in O_{\vartheta}(S)$.

Recall that $S$ is assumed to be finite. Thus, by Lemma 2.6, $O_{\vartheta}(S)$ is a closed subset of $S$. Note also that $O_{\vartheta}(S) \neq\{1\}$, since $s \neq 1$ and $s \in O_{\vartheta}(S)$. Thus, as $S$ is assumed to be primitive, $S=O_{\vartheta}(S)$, and that means that $S$ is thin.

An element $s$ of $S \backslash\{1\}$ will be called an involution if $\{1, s\}$ is closed. 
Lemma 4.7 Assume that $S$ is primitive and satisfies condition (a). Let $s$ be an element in $S \backslash\{1\}$, and assume that $s \in s s$ and $s^{*}=s$. Then $s$ is an involution and $S=\langle s\rangle$.

Proof We are assuming that $s^{*}=s$. Thus, by Lemma 4.4, $T_{s}=s s \backslash\{s\}$. It follows that $\left(T_{S}\right)^{*}=T_{s}$. Thus, by Lemma 2.5(ii), $T_{S}$ is closed. Thus, as $S$ is assumed to be primitive, $T_{s}=\{1\}$ or $T_{s}=S$.

Since $s \notin T_{s}, T_{s} \neq S$. It follows that $T_{s}=\{1\}$. Thus, as $T_{s}=s s \backslash\{s\}$, ss $=\{1\}$ or $s s=\{1, s\}$.

If $s s=\{1\}$, our hypothesis $s \in s s$ yields $s=1$. Since this contradicts our choice of $s$, we must have $s s=\{1, s\}$. Thus, $s$ is an involution. Thus, as $S$ is assumed to be primitive, $S=\langle s\rangle$.

An element $s$ of $S \backslash\{1\}$ will be called idempotent if $s s=\{s\}$ and $|\langle s\rangle|=3$.

Note that, for each idempotent element $s$ of $S,\langle s\rangle=\left\{1, s, s^{*}\right\}$.

Lemma 4.8 Assume that $S$ is primitive and satisfies condition (a). Let $s$ be an element in $S$, and assume $s \in$ ss and $s^{*} \neq s$. Then the following hold:

(i) We have $T_{s}=\{1, s\}$.

(ii) We have $s^{*} s=\left\{1, s, s^{*}\right\}$.

(iii) The element $s$ is idempotent, and $S=\langle s\rangle$.

Proof (i) We are assuming that $s \in s s$ and $s^{*} \neq s$. Thus, as $S$ is assumed to satisfy condition (a), $s s=\{s\}$. This means that $s \in T_{s}$. Thus, as $1 \in T_{s},\{1, s\} \subseteq T_{s}$.

Assume, by way of contradiction, that $T_{s} \nsubseteq\{1, s\}$. Then $T_{s}$ possesses an element $r$ different from 1 and $s$.

Since $r \in T_{s}, r \in s^{*} s \backslash\left\{s^{*}\right\}$; cf. Lemma 4.4. Thus, as $r \neq s, r \in s^{*} s \backslash\left\{s^{*}, s\right\}$. It follows that $r^{*} \in s^{*} \backslash \backslash\left\{s^{*}, s\right\} \subseteq T_{s}$. Thus, by Lemma 2.5(i), $\langle r\rangle \subseteq T_{s}$.

Now recall that we are assuming $S$ to be primitive. Thus, as $r \neq 1,\langle r\rangle=S$. It follows that $T_{s}=S$. This contradicts Lemma 4.4, where we saw that $s^{*} \notin T_{s}$.

(ii) Recall from Lemma 4.4 that $T_{s}=s^{*} s \backslash\left\{s^{*}\right\}$. Thus, by (i), $\{1, s\}=s^{*} s \backslash\left\{s^{*}\right\}$.

We are assuming that $s \in s s$. Thus, by H3, $s \in s s^{*}$. A second application of H3 now yields $s^{*} \in s^{*} s$. Thus, as $\{1, s\}=s^{*} s \backslash\left\{s^{*}\right\}$, we must have $s^{*} s=\left\{1, s, s^{*}\right\}$.

(iii) From $s^{*} \neq s$ we obtain $s \neq 1$. Thus, as $S$ is assumed to be primitive, $S=\langle s\rangle$.

We are assuming that $s \in s s$ and $s^{*} \neq s$. Thus, as $S$ is assumed to satisfy condition (a), $s s=\{s\}$.

From $s \in s s$ we also obtain $s^{*} \in s^{*} s^{*}$; cf. Lemma 1.3(ii). Thus, interchanging the roles of $s$ and $s^{*}$ in (ii) we obtain $s s^{*}=\left\{1, s, s^{*}\right\}$. Thus, $\langle s\rangle=\left\{1, s, s^{*}\right\}$.

We are now ready to prove Theorem A. For the convenience of the reader, we restate the theorem.

Theorem A Assume $S$ to be finite. Then the following conditions are equivalent:

(a) For any two elements $p$ and $q$ in $S$ with $p \neq q^{*}$, we have $|p q|=1$.

(b) The hypergroup $S$ is a wreath product of finitely many finite hypergroups all of which are thin or generated by an involution or by an idempotent element of $S$. 
Proof (a) $\Rightarrow$ (b) Since $S$ is assumed to be finite, we have $O_{\vartheta}(S) \subseteq T_{S}$ for each element $s$ in $S \backslash O_{\vartheta}(S)$; cf. Lemma 4.2. Thus, by definition,

$$
S \cong O_{\vartheta}(S)<S / / O_{\vartheta}(S)
$$

The fact that $O_{\vartheta}(S) \subseteq T_{S}$ for each element $s$ in $S \backslash O_{\vartheta}(S)$ also implies that $O_{\vartheta}(S)$ is normal in $S$; cf. Lemma 3.5. Thus, as $S$ is assumed to satisfy (a), $S / / O_{\vartheta}(S)$ satisfies (a); cf. Lemma 4.1.

Assume first that $O_{\vartheta}(S) \neq\{1\}$. Then, as $S / / O_{\vartheta}(S)$ satisfies (a), induction allows us to conclude that $S / / O_{\vartheta}(S)$ satisfies (b). Thus, as $S \cong O_{\vartheta}(S)$ ₹ $S / / O_{\vartheta}(S), S$ satisfies condition (b); cf. Theorem 3.6.

Assume now that $O_{\vartheta}(S)=\{1\}$. There is nothing to show if $S=\{1\}$. Thus, we assume that $S \neq\{1\}$. Among the closed subsets of $S$ different from $\{1\}$ we chose $T$ minimal. Note that this implies that $T$ is a primitive hypergroup satisfying (a).

Since $T \neq\{1\}, T \backslash\{1\}$ is not empty. We fix an element in $T \backslash\{1\}$ and call it $t$.

Since $O_{\vartheta}(S)=\{1\}, t \in t t$; cf. Lemma 4.6. Thus, by Lemmas 4.7 and 4.8(iii), $T=\langle t\rangle$, and $t$ is an involution or an idempotent element of $S$.

From Lemma 4.3(ii) we also obtain $T \subseteq T_{s}$ for each element $s$ in $S \backslash T$. Thus, by definition,

$$
S=T 2(S / / T) .
$$

As before, the fact that $T \subseteq T_{S}$ for each element $s$ in $S \backslash T$ also implies that $T$ is normal in $S$; cf. Lemma 3.5. Thus, as $S$ is assumed to satisfy (a), $S / / T$ satisfies (a); cf. Lemma 4.1.

Since $T \neq\{1\}$ and $S / / T$ satisfies (a), induction allows us to conclude that $S / / T$ satisfies (b). Thus, as $S \cong T$ 2 $S / / T, S$ satisfies condition (b); cf. Theorem 3.6.

(b) $\Rightarrow$ (a) Assume that there exist hypergroups $S_{1}, \ldots, S_{n}$ such that

$$
S=S_{1} \prec \cdots 2 S_{n}
$$

and, for each element $i$ in $\{1, \ldots, n\}, S_{i}$ is thin or generated by an involution or by an idempotent element of $S$. Clearly we may assume that $S_{i} \neq\{1\}$ for each element $i$ in $\{1, \ldots, n\}$.

Since $S=S_{1} 2 \cdots 2 S_{n}, S$ possesses a closed subset $T$ such that

$$
T \cong S_{1}, \quad S / / T \cong S_{2} 2 \cdots 2 S_{n},
$$

and $T \subseteq T_{S}$ for each element $s$ in $S \backslash T$. Note also that $T$ is normal in $S$; cf. Lemma 3.5.

Let $p$ and $q$ be elements in $S$ with $p \neq q^{*}$. We have to show that $|p q|=1$.

If $p, q \in T$, we obtain $|p q|=1$ from $T \cong S_{1}$ together with the fact that $S_{i}$ is thin or generated by an involution or by an idempotent element of $S$.

Assume that $p \in T$ and $q \notin T$. From $p \in T$ we obtain $p^{*} \in T$. From $q \notin T$ we obtain $q^{*} \notin T$, and that implies $T \subseteq T_{q^{*}}$. Thus, $p^{*} \in T_{q^{*}}$, and that means that $q^{*} p^{*}=$ $\left\{q^{*}\right\}$. Thus, by Lemma 1.3(ii), $p q=\{q\}$.

If $p \notin T$ and $q \in T$, we obtain $q \in T_{p}$, and that means that $p q=\{p\}$. 
Assume now that $p \notin T$ and $q \notin T$. Then $T p T=\{p\}$ and $T q T=\{q\}$. Thus, $T p T \neq T q^{*} T$, and that means that $p^{T} \neq\left(q^{T}\right)^{*}$. Thus, as $T \neq\{1\}$, induction yields

$$
p^{T} q^{T}=\left\{r^{T}\right\}
$$

for some element $r$ in $S$.

If $r \in T, T p T=T q^{*} T$; cf. Lemma 1.3(i). Thus, as $T p T=\{p\}$ and $T q T=\{q\}$, $p=q^{*}$. Since this contradicts our choice of $p$ and $q$, we conclude that $r \notin T$. Thus, $\operatorname{Tr} T=\{r\}$. Thus, as $p^{T} q^{T}=\left\{r^{T}\right\}, p q=\{r\}$.

\section{Proof of Theorem B, Part I}

In this short section, we deal with association schemes. We freely adopt the notation of [8].

Lemma 5.1 Idempotent elements of association schemes do not have finite valency.

Proof Let $S$ be an association scheme, let $s$ be an idempotent element of $S$, let $y$ be an element in $X$, and let $z$ be an element in $y s$.

Since $s$ is idempotent, $s s=\{s\}$. Thus, as $z \in y s, z s \subseteq y s s=y s$. Since $s \neq 1$, $z \notin z s$. Thus, $z s \subseteq y s \backslash\{z\}$. It follows that

$$
n_{s}=|z s| \leq|y s|-1=n_{s}-1,
$$

so that $n_{s}$ is not finite.

Proposition 5.2 Let $S$ be an association scheme on a finite set. Then the following conditions are equivalent:

(a) For any two elements $p$ and $q$ in $S$ with $p \neq q^{*}$, we have $|p q|=1$.

(b) The scheme $S$ is a wreath product of finitely many finite schemes all of which are thin or generated by an involution.

Proof Considering Theorem A, this follows immediately from Lemma 5.1.

\section{Proof of Theorem B, Part II}

In this section, the letter $X$ stands for a finite set, the letter $S$ for an association scheme on $X$. In our notation, we mostly follow [8].

Let $C$ denote the field of the complex numbers, $V$ the free $C$-module over $X$, and End $_{C}(V)$ the endomorphism ring of $V$. Then $C$ is a subring of the center of $\operatorname{End}_{C}(V)$.

For any subset $Q$ of $\operatorname{End}_{C}(V)$, we shall denote by $C[Q]$ the smallest subring of $\operatorname{End}_{C}(V)$ which contains $C$ and $Q$. 
Let $s$ be an element in $S$. Then, as $X$ is a basis of $V$, there exists a uniquely defined element $\sigma_{s}$ in $\operatorname{End}_{C}(V)$ such that

$$
x \sigma_{s}=\sum_{y \in x s} y
$$

for each element $x$ in $X$.

Note that $\left\{\sigma_{s} \mid s \in S\right\}$ is a linearly independent subset of $\operatorname{End}_{C}(V)$ and that

$$
\sigma_{p} \sigma_{q}=\sum_{r \in S} a_{p q r} \sigma_{r}
$$

for any two elements $p$ and $q$ in $S$. In particular, $\left\{\sigma_{s} \mid s \in S\right\}$ is a basis of the subring

$$
A:=C\left[\left\{\sigma_{s} \mid s \in S\right\}\right]
$$

of $\operatorname{End}_{C}(V) .^{5}$

For the remainder of this section, we fix an element in $X$ and call it $x$.

Let $s$ be an element in $S$. Then, as $X$ is a basis of $V$, there exists a uniquely defined element $\epsilon_{s}$ in $\operatorname{End}_{C}(V)$ such that

$$
y \epsilon_{s}= \begin{cases}y & \text { if }(x, y) \in s \\ 0 & \text { otherwise. }\end{cases}
$$

Note that $\left\{\epsilon_{s} \mid s \in S\right\}$ is a linearly independent subset of $\operatorname{End}_{C}(V)$ and that

$$
\epsilon_{p} \epsilon_{q}=\delta_{p q} \epsilon_{p}
$$

for any two elements $p$ and $q$ in $S$. In particular, $\left\{\epsilon_{S} \mid s \in S\right\}$ is a basis of the subring

$$
E:=C\left[\left\{\epsilon_{s} \mid s \in S\right\}\right]
$$

of $\operatorname{End}_{C}(V){ }^{6}$

We set

$$
T:=C\left[\left\{\sigma_{s} \mid s \in S\right\} \cup\left\{\epsilon_{s} \mid s \in S\right\}\right] .
$$

The ring $T$ was introduced in [7] and is known as the subconstituent algebra of $S$ with respect to $x$.

In [7, Lemma 3.4(i)] it was shown that $T$ is semisimple. It is easy to see and well known that each irreducible $T$-module arises as submodule of $V$ and that $T$ is generated as a ring by the elements $\epsilon_{p^{*}} \sigma_{r} \epsilon_{q}$ with $p, q, r \in S$. We shall refer to these facts at various instances without further mention.

In the following, we set

$$
\sigma:=\sum_{s \in S} \sigma_{s}
$$

and note that $\sigma \in A$.

\footnotetext{
${ }^{5}$ The algebra $A$ is often called the adjacency algebra of $S$ over $C$.

${ }^{6}$ The algebra $E$ is usually called the dual adjacency algebra of $S$ with respect to $x$. 
Lemma 6.1 Let $p, q$, and $r$ be elements in S. Then the following hold:

(i) We have $r \in p q$ if and only if $\epsilon_{p^{*}} \sigma_{r} \epsilon_{q} \neq 0$.

(ii) We have $p q=\{r\}$ if and only if $\epsilon_{p^{*}} \sigma_{r} \epsilon_{q}=\epsilon_{p^{*}} \sigma \epsilon_{q}$.

Proof (i) This follows immediately from the definition of $\epsilon_{p^{*}}, \sigma_{r}$, and $\epsilon_{q}$.

(ii) From (i) we obtain

$$
\epsilon_{p^{*}} \sigma \epsilon_{q}=\sum_{s \in S} \epsilon_{p^{*}} \sigma_{s} \epsilon_{q}=\sum_{r \in p q} \epsilon_{p^{*}} \sigma_{r} \epsilon_{q} .
$$

Thus, as $\left\{\epsilon_{p^{*}} \sigma_{r} \epsilon_{q} \mid r \in p q\right\}$ is linearly independent, $\epsilon_{p^{*}} \sigma_{r} \epsilon_{q}=\epsilon_{p^{*}} \sigma \epsilon_{q}$ if and only if $p q=\{r\}$.

Recall that condition (a) of Theorem B says that $|p q|=1$ for any two elements $p$ and $q$ in $S$ with $p \neq q^{*}$. For the remainder of this section, we shall always mean condition (a) of Theorem B if we speak about condition (a).

Lemma 6.2 Condition (a) holds if and only if $\epsilon_{p^{*}} \sigma_{r} \epsilon_{q}=\epsilon_{p^{*}} \sigma \epsilon_{q}$ for any three elements $p, q$, and $r$ in $S$ with $p \neq q^{*}$ and $r \in p q$.

Proof This follows immediately from Lemma 6.1(ii).

In the following, we set

$$
j:=\sum_{y \in X} y
$$

and note that $j \in V$.

We also notice that $j E$ is an irreducible $T$-module with basis $\left\{j \epsilon_{s} \mid s \in S\right\}$. The irreducible $T$-module $j E$ is called the primary module of $T$.

For each irreducible $T$-module $W$, we define

$$
\operatorname{Supp}(W):=\left\{s \in S \mid W \epsilon_{s} \neq\{0\}\right\}
$$

and note that $\operatorname{Supp}(j E)=S$.

Proposition 6.3 The following conditions are equivalent:

(a) For any two elements $p$ and $q$ in $S$ with $p \neq q^{*}$, we have $|p q|=1$.

(b) For each nonprimary irreducible $T$-module $W$, we have $|\operatorname{Supp}(W)|=1$.

Proof (a) $\Rightarrow$ (b) Let $W$ be an irreducible $T$-module different from $j E$. We may (and shall) assume that $W$ is a submodule of $V$.

Let $u$ be an element in $\operatorname{Supp}(W)$. Then, by definition, $W \epsilon_{u} \neq\{0\}$. We first claim that $W \epsilon_{u}$ is a $T$-module.

Note that, for each element $w$ in $W, w \in W \epsilon_{u}$ if and only if $w \epsilon_{u}=w$.

Let $w$ be an element in $W \epsilon_{u}$, and let $p, q$, and $r$ be elements in $S$. Then, as $T$ is generated as a ring by the elements $\epsilon_{p *} \sigma_{r} \epsilon_{q}$ with $p, q, r \in S$, it suffices to show that $w \epsilon_{p^{*}} \sigma_{r} \epsilon_{q} \in W \epsilon_{u}$. 
If $u \neq p^{*}$, we have $w \epsilon_{p} \sigma_{r} \epsilon_{q}=w \epsilon_{u} \epsilon_{p} \sigma_{r} \epsilon_{q}=0$, and we are done. Thus, we may assume that $u=p^{*}$. By Lemma 6.1(i), we may also assume that $r \in p q$.

Assume that $u \neq q$. Then, as $u=p^{*}$ and $r \in p q, \epsilon_{p^{*} \sigma_{r}} \epsilon_{q}=\epsilon_{p^{*} \sigma} \sigma \epsilon_{q}$; cf. Lemma 6.2. Now recall that $w\left(\epsilon_{p^{*}} \sigma\right) \in W$ and that $\left(w \epsilon_{p^{*}}\right) \sigma \in j E$. Thus, as $W \neq j E$, $w \epsilon_{p *} \sigma=0$. It follows that $w \epsilon_{p^{*}} \sigma_{r} \epsilon_{q}=w \epsilon_{p^{*}} \sigma \epsilon_{q}=0$, and we are done.

Assume that $u=q$. Then $w \epsilon_{p^{*}} \sigma_{r} \epsilon_{q}=\left(w \epsilon_{p^{*}} \sigma_{r} \epsilon_{q}\right) \epsilon_{u}$, so that $w \epsilon_{p^{*}} \sigma_{r} \epsilon_{q} \in W \epsilon_{u}$.

We have seen that $W \epsilon_{u}$ is a $T$-module. Thus, as $W \epsilon_{u} \neq\{0\}$, the irreducibility of $W$ forces $W \epsilon_{u}=W$. It follows that $W \epsilon_{s}=\{0\}$ for each element $s$ in $S \backslash\{u\}$, and that means that $\operatorname{Supp}(W)=\{u\}$.

(b) $\Rightarrow$ (a) Let $p$ and $q$ be elements of $S$ with $p \neq q^{*}$, and let $r$ be an element in $p q$. We have to show that $p q=\{r\}$.

Let $W$ be an irreducible $T$-module different from $j E$. Thus, as $p \neq q^{*}$, we have $W \epsilon_{p^{*}}=\{0\}$ or $W \epsilon_{q}=\{0\}$. It follows that

$$
W \epsilon_{p^{*}} \sigma_{t} \epsilon_{q}=\{0\}
$$

for each element $t$ in $S$. As a consequence,

$$
W \epsilon_{p^{*}} \sigma \epsilon_{q}=\{0\}
$$

Now we determine the action of $\epsilon_{p^{*}} \sigma_{r} \epsilon_{q}$ on the $T$-submodule $j E$ of $V$. Recall that $\left\{j \epsilon_{s} \mid s \in S\right\}$ is a basis of $j E$. For each element $s$ in $S$, we have

$$
\left(j \epsilon_{s}\right)\left(\epsilon_{p^{*}} \sigma_{r} \epsilon_{q}\right)=\delta_{s p^{*}} j \epsilon_{p^{*}} \sigma_{r} \epsilon_{q}=\delta_{s p^{*}} a_{p^{*} r q} j \epsilon_{q}
$$

Thus, as $\sigma$ is defined to be the sum of the endomorphisms $\sigma_{s}$ with $s \in S$, we obtain from [8, Lemma 1.1.3(iii)] that

$$
\left(j \epsilon_{s}\right)\left(\epsilon_{p^{*}} \sigma \epsilon_{q}\right)=\delta_{s p^{*}} n_{p^{*}} j \epsilon_{q}
$$

for each element $s$ in $S$.

Thus,

$$
\epsilon_{p^{*}} \sigma \epsilon_{q}=\frac{n_{p^{*}}}{a_{p^{*} r q}} \epsilon_{p^{*}} \sigma_{r} \epsilon_{q}
$$

On the other hand,

$$
\epsilon_{p^{*}} \sigma \epsilon_{q}=\epsilon_{p^{*}} \sum_{s \in S} \sigma_{s} \epsilon_{q}=\sum_{s \in S} \epsilon_{p^{*}} \sigma_{s} \epsilon_{q}=\sum_{s \in p q} \epsilon_{p^{*}} \sigma_{s} \epsilon_{q}
$$

Thus, as $\left\{\epsilon_{p^{*}} \sigma_{s} \epsilon_{q} \mid s \in p q\right\}$ is a linearly independent subset of $\operatorname{End}(V)$, we must have $p q=\{r\}$.

We conclude this section by mentioning that Theorem $\mathrm{B}$ is a consequence of Propositions 5.2 and 6.3. 


\section{References}

1. Blau, H.: Table algebras. Eur. J. Comb. 30, 1426-1455 (2009)

2. Blau, H., Zieschang, P.-H.: Sylow theory for table algebras, fusion rule algebras, and hypergroups. J. Algebra 273, 551-570 (2004)

3. Bloom, W., Heyer, H.: Harmonic Analysis of Probability Measures on Hypergroups. de Gruyter, Berlin (1995)

4. Marty, F.: Sur une généralisation de la notion de groupe. In: Huitième Congres des Mathématiciens. Stockholm, pp. 45-59 (1934)

5. Song, S.-Y.: Fusion relation in products of association schemes. Graphs Comb. 18, 655-665 (2002)

6. Tanaka, R.: Classification of commutative association schemes with almost commutative Terwilliger algebras. J. Algebr. Comb. 33, 1-10 (2011)

7. Terwilliger, P.: The subconstituent algebra of an association scheme, (part I). J. Algebr. Comb. 1, 363388 (1992)

8. Zieschang, P.-H.: Theory of Association Schemes. Springer Monographs in Mathematics. Springer, Berlin (2005)

9. Zieschang, P.-H.: Hypergroups. Preprint, Max-Planck-Institut für Mathematik, Preprint Series 2010 (97) 